19,05

\title{
Тепловые, магнитные и магнитотранспортные свойства быстрозакаленнго ленточного образца $\mathrm{Ni}_{50} \mathrm{Mn}_{35} \mathrm{Al}_{2} \mathrm{Sn}_{13}$
}

\author{
(c) Ш.К. Хизриев ${ }^{1}$, А.Г. Гамзатов ${ }^{1}$, А.Б. Батдалов ${ }^{1}$, А.М. Алиев ${ }^{1}$, Л.Н. Ханов ${ }^{1}$, D.-Н. Kim $^{2}$, \\ S.-C. $Y u^{2}$, N.H. Yen ${ }^{3}$, N.H. $\operatorname{Dan}^{3}$ \\ ${ }^{1}$ Институт фризики им. Х.И. Амирханова, ДФИЦ РАН, \\ Махачкала, Россия \\ ${ }^{2}$ Department of Physics, Chungbuk National University, \\ Cheongju, 28644, South Korea \\ ${ }^{3}$ Institute of Materials Science, VAST, 18-Hoang Quoc Viet, \\ Hanoi, Vietnam \\ E-mail: Shakhban1994@gmail.com
}

Поступила в Редакцию 10 февраля 2020 г.

В окончательной редакции 3 марта 2020 г.

Принята к публикации 10 марта 2020 г.

\begin{abstract}
Исследованы теплоемкость, температурапроводность, теплопроводность, намагниченность и электросопротивление быстрозакаленного сплава $\mathrm{Ni}_{50} \mathrm{Mn}_{35} \mathrm{Al}_{2} \mathrm{Sn}_{13}$. На температурной зависимости температурапроводности наблюдаются ярко выраженные аномалии в виде минимумов, связанные с магнитным и магнитоструктурными превращениями. Поведение теплопроводности и температурапроводности указывает на то, что длина свободного пробега носителей тепла ограничена структурными несовершенствами ленты. Переход образца в мартенситную фазу сопровождается резким ростом электросопротивления, предполагающий усиление скорости релаксации электронов на структурных искажениях, присущих мартенситу. Величина магниторезистивного эффекта $\Delta \rho / \rho_{0}$ в поле $1.8 \mathrm{~T}$ вблизи мартенситного перехода достигает $40 \%$. На зависимости $\rho(T)$ вблизи $T_{C}$ обнаружен температурный гистерезис, свидетельствующий о структурной неоднородности аустенитной фазы.
\end{abstract}

Ключевые слова: теплоемкость, электросопротивление, намагниченность, температурапроводность, теплопроводность.

DOI: 10.21883/FTT.2020.07.49486.025

\section{1. Введение}

Сплавы Гейслера являются предметом пристального внимания исследователей, так как на основе их физических свойств могут быть созданы новые инновационные технологии

В образцах базовой композиции $\mathrm{Ni}_{50} \mathrm{Mn}_{37} \mathrm{Sn}_{13}$ при понижении температуры наблюдаются сначала магнитный (парамагнетик-ферромагнетик), а затем и структурный (аустенит-мартенсит) фазовые переходы. Структурный фазовый переход, как правило, сопровождается переходом из ферромагнитного состояния в антиферромагнитное. Результаты исследования магнитных, тепловых и магнитокалорических свойств массивных образцов сплава $\mathrm{Ni}_{50} \mathrm{Mn}_{37} \mathrm{Sn}_{13}$ приведены в работах [1-5].

Характерной особенностью данной композиции является незначительное различие в значениях критических температур: за магнитным фазовым переходом в аустенитной фазе $\left(T_{C}\right)$ почти сразу начинается структурный переход аустенит-мартенсит. Так, по данным [4] $T_{C}=310 \mathrm{~K}$, а $M_{S}$ (температура начала мартенситного перехода) $=305 \mathrm{~K}$, а согласно [2] $T_{C}=311 \mathrm{~K}$, $M_{S}=307 \mathrm{~K}$. Последствием такой близости может стать более сильная взаимосвязь магнитной и структурной подсистемы, и наблюдаемые нетривиальные физические свойства сплава $\mathrm{Ni}_{50} \mathrm{Mn}_{37} \mathrm{Sn}_{13}$.
Сведение о магнитных, структурных и магнитокалорических свойствах быстрозакаленного базового состава $\mathrm{Ni}_{50} \mathrm{Mn}_{37} \mathrm{Sn}_{13}$ приводятся в работах [6-10]. Анализ полученных данных показывает, что быстрозакаленные образцы полностью сохраняют структурные и магнитные фазовые превращения, присущие объемным образцам. С технологической точки зрения быстрозакаленные образцы имеют некоторые преимущества перед массивными, так как позволяют изготавливать изделия любой конфигурации. Это преимущество особенно важно для технологии магнитного охлаждения, когда необходимо максимально увеличивать скорость отвода тепла от рабочего тела холодильной машины.

Управлять магнитными, магнитокалорическими и магнитотранспортными свойствами ферромагнитных сплавов Гейслера можно как путем изменения соотношения компонент исходного состава $\mathrm{Ni}-\mathrm{Mn}-\mathrm{Sn}$, так и путем замещения элементов базового состава другими металлами. Некоторые сведения о влиянии замещения атомов исходного состава атомами других элементов на физические свойства массивных образцов сплава $\mathrm{Ni}-\mathrm{Mn}-\mathrm{Sn}$ приведены в [11-15].

Привлекаются и другие технологические приемы для улучшения свойств сплавов, в частности, отжиг при высоких температурах, который снимает механические 
напряжения, особенно присущие быстрозакаленным образцам и способствует магнитному и структурному упорядочению и существенно улучшает их магнитотранспортные характеристики [9,16-19]. Так, согласно $[9,17]$ отжиг пленок $\mathrm{Ni}_{50} \mathrm{Mn}_{37} \mathrm{Sn}_{13}$ при высоких температурах приводит к многократному росту изменения энтропии $\Delta S_{m}$ и к сдвигу максимума магнитокалорического эффекта в сторону высоких температур.

В то же время вопросы, связанные с влиянием микроструктуры, получаемой при быстрой закалке на магнитотранспортные, теплофизические и магнитокалорические свойства лент пока не нашли должного отражения в литературе, хотя актуальность таких исследовании очевидна.

В настоящем сообщении приводятся результаты исследования магнитных, теплофизических и магнитотранспортных свойств быстрозакаленного ленточного образца $\mathrm{Ni}_{50} \mathrm{Mn}_{37} \mathrm{Al}_{2} \mathrm{Sn}_{13}$ в области $T=77-350 \mathrm{~K}$ и в магнитном поле $1.8 \mathrm{~T}$.

\section{2. Образцы и методика}

Исследованные ленты были получены методом быстрой закалки из расплава (спинингование). Ширина ленты $\approx 4 \mathrm{~mm}$, толщина $\approx 30 \mu \mathrm{m}$. Рентгеноструктурный анализ показал, что образцы являются однофазными, а аустенит имеет кубическую структуру типа L $2{ }_{1}$. Тонкости технологии получения и некоторые физические свойства лент $\mathrm{Ni}_{50} \mathrm{Mn}_{37-x} \mathrm{Al}_{x} \mathrm{Sn}_{13}$, исследуемых в настоящей работе приведены в работе [20].

Изображение микроструктуры поверхности ленты получено методом электронной микроскопии и приведено на рис. 1. Четко видна зернистая структура поверхности

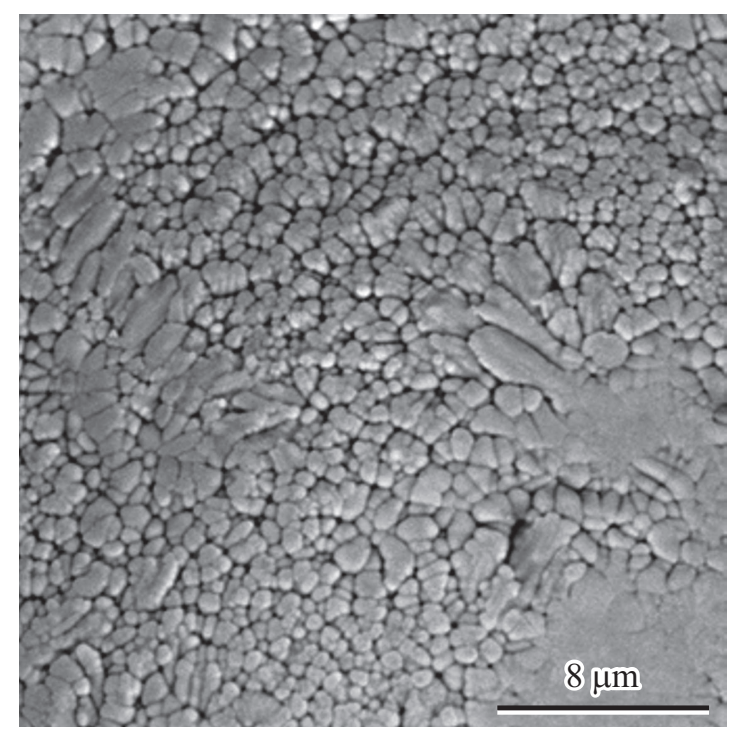

Рис. 1. Микроструктура поверхности ленты, полученная методом электронной микроскопии. Четко видна зернистая структура поверхности с размерами зерна около $1 \mu \mathrm{m}$, между которыми имеется некоторое свободное пространство. с размерами зерна около $1 \mu \mathrm{m}$, между которыми имеется некоторое свободное пространство. Наблюдаются области с разным распределением гранул по размерам на поверхности ленты, т. е. лента неоднородна по структуре.

Теплоемкость $C_{P}$ и температурапроводность $\eta$ образца измерялись методом ас-калориметрии, теплопроводность $\kappa$ оценивалась с помощью соотношения: $\kappa=\frac{d}{M} C_{p} \eta$ (где $d$ и $M-$ плотность и молярная масса образца соответственно) электросопротивление четырехконтактным методом, намагниченность - на вибрационном магнитометре.

\section{3. Результаты и обсуждение}

Как показали авторы [21,22] частичные замещения атомов $\mathrm{Ni}$ атомами Со в образцах $\mathrm{Ni}_{50-x} \mathrm{Co}_{x} \mathrm{Mn}_{37} \mathrm{Sn}_{13}$ $(x=1,2,3)$ приводит к улучшению магнитных характеристик лент, росту $T_{C}$ и уменьшению температуры структурных превращений.

В [23] анализируется влияние замещения $\mathrm{Ni}$ одновалентным $\mathrm{Ag}$ на свойства лент $\mathrm{Ni}_{50-x} \mathrm{Ag}_{x} \mathrm{Mn}_{37} \mathrm{Sn}_{13}$ $(x=1,2,4)$. Основным результатом данного исследования является то, что такое замещение приводит к систематическому уменьшению как $T_{C}$, так и $T_{S}$, а в композиции с $x=4$ мартенситный переход вообще не наблюдается.

Для анализа влияния различного рода замещений на физические свойства сплавов Гейслера часто пользуются такой характеристикой, как средневзвешенная концентрация валентных электронов на атом (e/a). Известно, что свойства сплавов сильно зависят от этой величины: рост отношения (e/a) сопровождается увеличением температуры магнитоструктурных превращении $T_{S}[24,25]$. Замещение части атомов $\mathrm{Mn}$ атомами $\mathrm{Al}$ в сплаве $\mathrm{Ni}_{50} \mathrm{Mn}_{37-x} \mathrm{Al}_{x} \mathrm{Sn}_{13}$ уменьшает указанное отношение от $8.11(x=0)$ до $8.03(x=2)$ и вызывает уменьшение температуры перехода аустенит-мартенсит от $307 \mathrm{~K}(x=0)$ до $172 \mathrm{~K}(x=2)$. В то же время такое замещение усиливает обменные взаимодействия между атомами $\mathrm{Mn}$ и $\mathrm{Ni}$ и приводит к незначительному росту $T_{C}$.

Данные по температурной зависимости намагниченности лент (рис. 2) показывают, что исследуемый образец при понижении температуры испытывает как магнитный (при $T_{C}=315 \mathrm{~K}$ ), так и магнитоструктурный (при $\left.T_{S}=175 \mathrm{~K}\right)$ фазовые переходы.

Использованная нами для измерения теплоемкости $C_{p}(T)$ методика позволяет с большой точностью проследить температурный ход $C_{p}(T)$ для образцов малых размеров. Результаты измерения теплоемкости приведена на рис. 3. Резкие скачки, характерные для массивных образцов вблизи температур фазовых переходов не наблюдаются, однако видны небольшие „горбики“ вблизи магнитоструктурного $\left(T_{S}=170 \mathrm{~K}\right)$ и магнитного $\left(T_{C}=310 \mathrm{~K}\right)$ переходов. В то же время для ленточных образцов базовой композиции $\mathrm{Ni}_{50} \mathrm{Mn}_{37} \mathrm{Sn}_{13}$ такие 


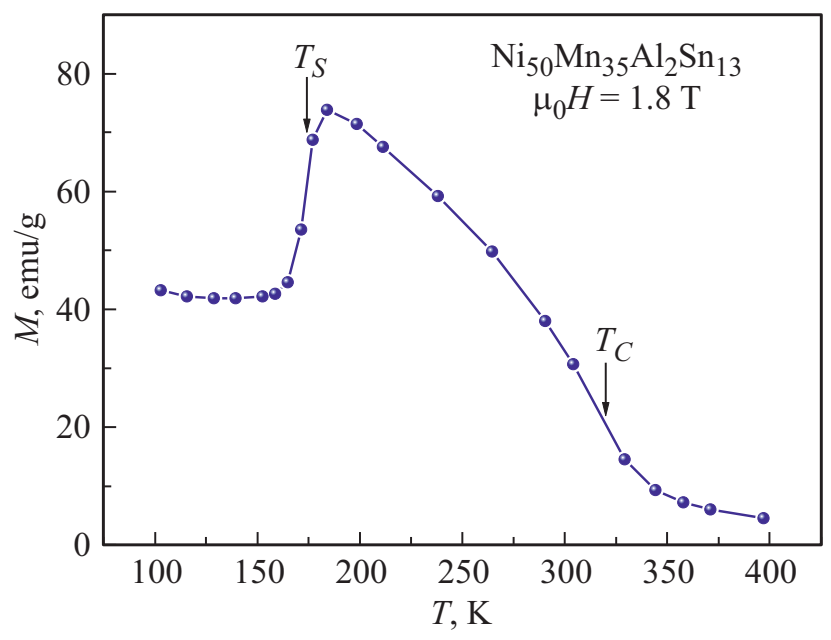

Рис. 2. Зависимость намагниченности от температуры.

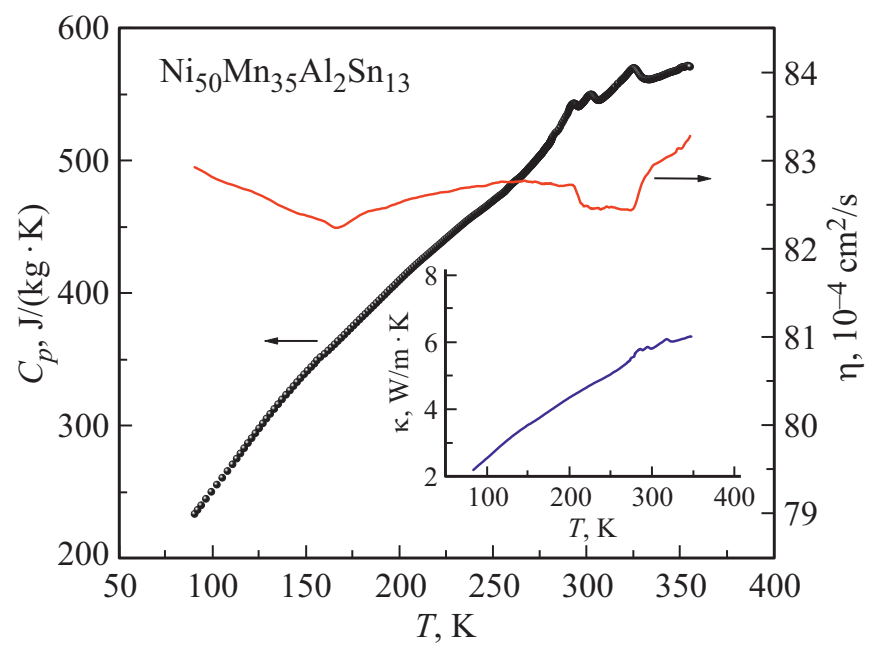

Рис. 3. Зависимость теплоемкости и температуропроводности от температуры. На вставке - температурная зависимость теплопроводности.

аномалии четко наблюдаются [8]. В качестве причин такого поведения $C_{p}(T)$ можно привести возможные структурные искажения и химическую неоднородность исследованных лент, приводящих к размытию фазовых переходов. Следует так же отметить более крутой, чем для массивных образцов и растянутый по температуре почти линейный рост теплоемкости с температурой для быстрозакаленного образца (см. рис. 3 ).

На рис. 3 также приведены данные для температурной зависимости температурапроводности и теплопроводности в интервале температур 80-350 К. Следует заметить, что на всем температурном интервале $(80-350 \mathrm{~K})$ величина температурапроводности слабо меняется. Это скорее всего связано с ярко выраженными неоднородностями микроструктуры лент, которые приводят к слабому изменению механизмов рассеяния носителей тепла в рассматриваемом интервале температур. Однако на температурной зависимости температурапроводности наблюдается аномалии в виде минимумов вблизи магнитного и магнитоструктурного фазовых переходов, связанные с критическим рассеянием носителей тепла на искажениях кристаллической структуры и на флуктуациях магнитного параметра порядка.

Данные по теплоемкости $C_{p}(T)$ и термодиффузии $\eta(T)$ можно использовать для вычисления теплопроводности, так как они связаны соотношением $\kappa=\frac{d}{M} C_{p} \eta$. Полученная таким образом зависимость $\kappa(T)$ приведена на вставке рис. 3. Как видно, температурная зависимость теплопроводности определяется температурным ходом теплоемкости, так как на всем интервале температур температурапроводность слабо меняется. По выше приведенным причинам мы не наблюдаем резких аномалий в поведении $\kappa(T)$, характерных для массивных образцов сплавов вблизи критических температур [26]. Величина теплопроводности несколько меньше чем для массивных образцов сплава Гейслера [26-28], а электронная составляющая, оцененная по данным $\rho(T)$ из соотношения Видемана-Франца $k_{e}=L_{0} \frac{T}{\rho}\left(L_{0}-\right.$ число Лоренца $)$ почти на два порядка меньше, чем экспериментальная величина теплопроводности. Это означает, что теплоперенос в основном является фононным.

Температурная зависимость электросопротивления $\rho(T)$ имеет вид, характерный для сплавов Гейслера с магнитоструктурными фазовыми переходами (рис. 4). C ростом температуры вблизи перехода мартенситаустенит сопротивление резко падает, что обычно связывается с переходом образца из низкосимметричной модулированной мартенситной фазы в высокосимметричный кубическии аустенит со структурой L2 1 [21,29,30]. Данное утверждение основано на том, что значительный вклад в рассеяние электронов вносят мелкомасштабные структурные искажения, которые характерны для мартенсита и исчезают при переходе в ферромагнитный аустенит.

Однако существует и другое мнение по этому поводу. Авторы [18] на основе изучения магнитосопротивления ленточных образцов сплава $\mathrm{Ni}_{44.1} \mathrm{Mn}_{44.2} \mathrm{Sn}_{11.7}$ приходят к выводу, что аномалии в поведении $\rho(T)$ при переходе в аустенитную фазу связаны с изменениями в электронном спектре сплава, т.е. с изменениями плотности электронных состоянии вблизи поверхности Ферми. Ясность в обсуждаемый вопрос могли бы внести измерения нормального коэффициента Холла в мартенситной и аустенитной фазе.

Сравнение результатов наших исследовании с литературными данными для массивных образцов показывает, что дефекты в виде структурных неоднородностей и локальных деформаций, возникающих в процессе спинингования оказывают более существенное влияние на электроперенос и не столь критичны для теплопереноса.

В области магнитного фазового перехода наблюдается и не характерный для таких переходов температурный гистерезис в поведении $\rho(T)$, который обычно связывается со структурными превращениями. Возможно, 


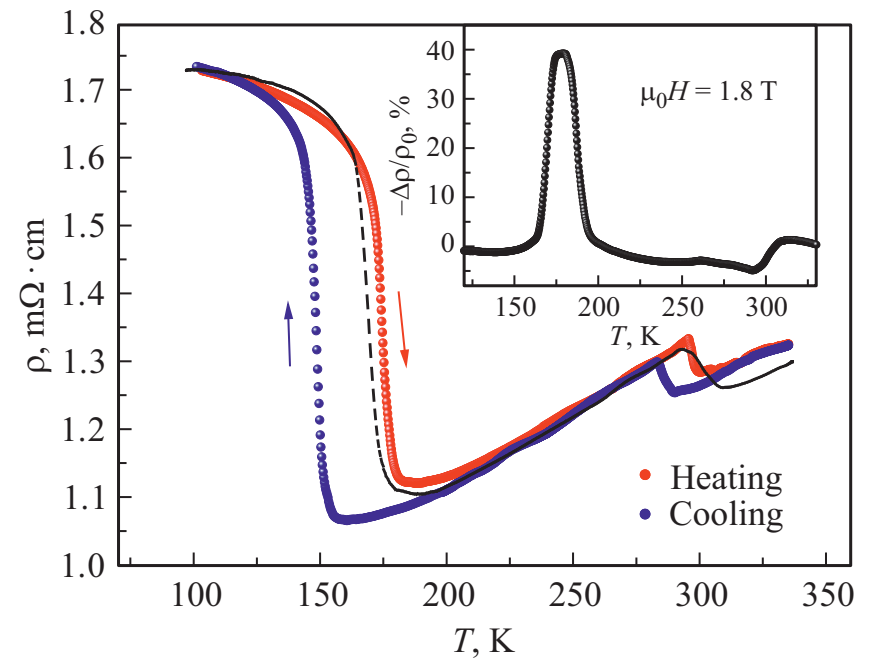

Рис. 4. Температурная зависимость электросопротивления в режиме нагрева и охлаждения. Пунктирная линия соответствует $\rho(T)$ в поле $1.8 \mathrm{~T}$ в режиме нагрева. На вставке магнитосопротивление в зависимости от температуры.

наблюдаемое при $T_{C}$ резкое падение электросопротивления, и температурный гистерезис, связаны с исчезновением остатков мартенситной фазы с другой критической температурой.

Приложение магнитного поля стабилизирует высокотемпературную аустенитную фазу и приводит к сдвигу кривой $\rho(T)$ в сторону низких температур и появлению отрицательного магнитосопротивления, которое в данном случае достигает $40 \%$ в поле $1.8 \mathrm{~T}$ (см. вставку на рис. 4).

\section{4. Заключение}

Таким образом проведены исследования намагниченности, теплоемкости, температурапроводности, теплопроводности, электросопротивления быстрозакаленного ленточного образца сплава $\mathrm{Ni}_{50} \mathrm{Mn}_{35} \mathrm{Al}_{2} \mathrm{Sn}_{13}$ в области $T=80-350 \mathrm{~K}$ и в магнитном поле $1.8 \mathrm{~T}$. В поведении исследуемых коэффициентов вблизи критических температур проявляются аномалии, присущие и массивным образцам сплавов. Микроструктура, полученная при спининговании эффективно рассеивает электроны проводимости и слабо влияет на теплоперенос.

Наблюдаемая слабая зависимость температурапроводности от температуры на всем интервале $(80-350 \mathrm{~K})$ скорее всего связана с ярко выраженными неоднородностями микроструктуры лент, которые приводят к слабому изменению механизмов рассеяния носителей тепла.

На зависимости $\rho(T)$ вблизи $T_{C}$ наблюдается температурный гистерезис, указывающий на структурную неоднородность аустенитной фазы. Величина магниторезистивного эффекта в поле 1.8 Т достигает $40 \%$.

\section{Финансирование работы}

Работа выполнена при финансовой поддержке гранта РФФИ № 19-08-00782, а также в рамках государственного задания № АААА - A17-117021310366-5.

\section{Конфликт интересов}

Авторы заявляют, что у них нет конфликта интересов.

\section{Список литературы}

[1] Y. Sutou, Y. Imano, N. Koeda, T. Omori, R. Kainuma, K. Ishida, K. Oikawa. Appl. Phys. Lett. 85, 4358 (2004).

[2] T. Krenke, E. Duman, M. Acet, E.F. Wassermann, X. Moya, L. Manosa, A. Planes. Nature Mater. 4, 450 (2005).

[3] Ю.В. Калетина, Е.Г. Герасимов, В.А. Казанцев, А.Ю. Калетин. ФТТ 10, 1978 (2017).

[4] S. Aksoy, M. Acet, P.P. Deen, L. Mañosa, A. Planes. Phys. Rev. B 79, 212401 (2009).

[5] T. Krenke, M. Acet, E. Wassermann, X. Moya, L. Mañosa, A. Planes. Phys. Rev. B 72, 014412 (2005).

[6] T.L. Phan, P. Zhang, N.H. Dan, N.H. Yen, P.T. Thanh, T.D. Thanh, M.H. Phan, S.C. Yu. Appl. Phys. Lett. 101, 212403 (2012).

[7] R. Caballero-Flores, L. González-Legarreta, W.O. Rosa, T. Sánchez, V.M. Prida, Ll. Escoda, J.J. Suñol, A.B. Batdalov, A.M. Aliev, V.V. Koledov, V.G. Shavrov, B. Hernando. J. Alloys. Compd. 629, 332 (2015).

[8] A.M. Aliev, A.B. Batdalov, I.K. Kamilov, V.V. Koledov, V.G. Shavrov, V.D. Buchelnikov, J. Garcia, V.M. Prida, B. Hernando. Appl. Phys. Lett. 97, 212505 (2010).

[9] D. Wu, S. Xue, J. Frenzel, G. Eggeler, Q. Zhai, H. Zheng. Mater. Sci. Eng. A 534, 568 (2012).

[10] Y.B. Yang, X.B. Ma, X.G. Chen, J.Z. Wei, R. Wu, J.Z. Han, H.L. Du, C.S. Wang, S.Q. Liu, Y.C. Yang, Y. Zhang, J.B. Yang. J. Appl. Phys. 111, 07A916 (2012).

[11] A. Banerjee, P. Chaddah, S. Dash, K. Kumar, A. Lakhani. Phys. Rev. B 84, 214420 (2011).

[12] R. Das, S. Sarma, A. Perumal, A. Srinivasan. J. Appl. Phys. 109, 07A901 (2011).

[13] S. Pramanick, S. Chatterjee, S. Giri, S. Majumdar. Appl. Phys. Lett. 105, 112407 (2014).

[14] V.K. Sharma, M.K. Chattopadhyay, R. Kumar, T. Ganguli, P. Tiwari, S.B. Roy. J. Phys.: Condens. Matter. 19, 496207 (2007).

[15] S.M. Podgornykh, E.G. Gerasimov, N.V. Mushnikov, T. Kanomata. J. Phys.: Conf. Ser. 266, 012004 (2011).

[16] A. Quintana-Nedelcos, J.L. Sanchez Llamazares, G. DanielPerez. J. Magn. Magn. Mater. 441, 188 (2017).

[17] W. Wang, H. Li, J. Ren, J. Fu, Q. Zhai, Z. Luo, H. Zheng. J. Magn. Magn. Mater. 374, 153 (2015).

[18] H.C. Xuan, Y. Deng, D.H. Wang, C.L. Zhang, Z.D. Han, Y.W. Du. J. Phys. D 41, 215002 (2008).

[19] B. Weise, B. Dutta, N. Teichert, A. Hutten, T. Hickel, A. Waske. Sci. Rep. 8, 9147 (2018).

[20] H.Y. Nguyen, T.M. Nguyen, M.Q. Vu, T.T. Pham, D.T. Tran, H.D. Nguyen, L.T. Nguyen, H.H. Nguyen, V. Koledov, A. Kamantsev, A. Mashirov, H.D. Nguyen. Adv. Nature Sci: Nanosci. Nanotechnol. 9, 025007 (2018). 
[21] L. Chen, F.X. Hu, J. Wang, L.F. Bao, X.Q. Zheng, L.Q. Pan, J.H. Yin, J.R. Sun, B.G. Shen. J. Alloys. Compd. 549, 170 (2013).

[22] S. Louidi, J.J. Sunol, M. Ipatov, B. Hernando. J. Alloys. Compd. 739, 305 (2018).

[23] T.D. Thanh, N.H. Duc, N.H. Dan, N.T. Mai, T.L. Phan, S.K. Oh, S.C. Yu. J. Alloys. Compd. 696, 1129 (2017).

[24] A. Planes, L. Mañosa, M. Acet. J. Phys.: Condens. Matter. 21, 233201 (2009).

[25] T. Krenke, X. Moya, S. Aksoy, M. Acet, P. Entel, Ll. Manosa, A. Planes, Y. Elerman, A. Yucel, E.F. Wassermann. J. Magn. Magn. Mater. 310, 2788 (2007).

[26] Y.K. Kuo, K.M. Sivakumar, H.C. Chen, J.H. Su, C.S. Sue. Phys. Rev. B 72, 054116 (2005).

[27] P. Czaja, J. Przewoźnik, Ł. Gondek, L. Hawelek, A. Żywczak, E. Zschech. J. Magn. Magn. Mater. 421, 19 (2017).

[28] M. Seredina, M. Lyange, V. Khovaylo, S. Taskaev, H. Miki, T. Takagi, R. Singh, R. Chatterjee, L.K. Varga. Mater. Sci. Forum 845, 65 (2016).

Редактор Т.Н. Василевская 